\title{
CORRELAÇÃO LINEAR E ESPACIAL ENTRE A PRODUTIVIDADE DE FORRAGEM DE MILHO E FRAÇÕES GRANULOMÉTRICAS DE UM LATOSSOLO VERMELHO DISTRÓFICO ${ }^{(1)}$
}

\author{
CÉSAR GUSTAVO DA ROCHA LIMA $\left({ }^{2}\right)$; MOREL DE PASSOS E CARVALHO $\left(3^{*}\right)$
}

\begin{abstract}
RESUMO
A distribuição granulométrica do solo, por possuir estreita relação com a capacidade de retenção e disponibilidade de água e nutrientes, é um fator de grande influência na produtividade vegetal. O objetivo foi estudar a variabilidade e as correlações lineares e espaciais entre os atributos da planta e do solo. Foi instalada uma malha de amostragem, para a coleta de dados do solo e planta, contendo 125 pontos, em uma área de $2500 \mathrm{~m}^{2}$, no ano agrícola de 2005, no município de Pereira Barreto (SP). Foram analisadas a produtividade de forragem do milho outonal (MSF) no sistema plantio direto irrigado e as frações granulométricas areia (AR), silte (SI) e argila (AG) em profundidade, em Latossolo Vermelho Distrófico. Nos atributos estudados, observou-se variabilidade dos dados entre média e baixa, e espaciais não variaram aleatoriamente, seguindo padrões bem definidos, com alcances entre 8,5 e 46,8 metros. Não houve correlação linear consistente entre o atributo da planta com os do solo, porém houve uma apreciável correlação espacial entre a MSF e a argila de 0,20-0,30 m (AG3).
\end{abstract}

Palavras-chave: atributos físicos do solo, granulometria do solo, plantio direto, forragicultura.

\section{ABSTRACT \\ LINEAR AND SPATIAL CORRELATION BETWEEN CORN YIELD AND PARTICLE-SIZE DISTRIBUTION IN A HAPLIC ACRUSTOX}

The distribution soil particle-size has a narrow relationship with the nutrients and water holding capacity and influences plant yield. The purpose was to study the variability and linear and spatial correlations among plant and soil attributes. A sampling grid to collect soil and plant data was installed with one hundred and twenty five sampling points in an area of $2500 \mathrm{~m}^{2}$. The forage yield of autumnal corn (CPF) in no-tillage and irrigated systems, the contents of soil sand (SA), silt (SI), and clay (CL), at the depths $1(0,00-0,10 \mathrm{~m}), 2(0,10-0,20 \mathrm{~m})$, and $3(0,20-0,30 \mathrm{~m})$, in a Haplic Acrustox of Bonança Farm at Pereira Barreto County, Sao Paulo State, Brazil, were analyzed in the agricultural year of 2005. The studied attributes showed median to low variability, with excellent spatial performances, and ranges between 8,5 and 46,8 meters. Oppositely, the linear correlations between CPF and soil attributes were low. There was appreciable spatial correlation between CPF and clay of the 0,20-0,30 m (CL3).

Key words: soil physical attributes, particle-size distribution, no-tillage, crop forage.

( $\left.{ }^{1}\right)$ Parte da dissertação de mestrado do Programa de Pós-Graduação em Sistemas de Produção - Faculdade de Engenharia UNESP/Campus de Ilha Solteira. Recebido para publicação em 20 de agosto de 2007 e aceito em 30 de abril de 2009.

$\left(^{2}\right)$ Mestrando da Faculdade de Engenharia - UNESP/Campus de Ilha Solteira. E-mail: etaugustus@aluno.feis.unesp.br

$\left({ }^{3}\right)$ Departamento de Fitossanidade, Engenharia Rural e Solos - Faculdade de Engenharia - UNESP/Campus de Ilha Solteira. Caixa Postal 31, 15385-000 Ilha Solteira (SP). E-mail: morel@agr.feis.unesp.br; mpcarva53@yahoo.com.br $\left({ }^{*}\right)$ Autor correspondente. 
O milho é um dos mais importantes cereais cultivados e consumidos no mundo. O Brasil tem-se destacado como o terceiro maior produtor desse grão, com a safra recorde, em 2001, de 41,5 milhões de toneladas (EMBrapa, 2006a), porém baixa produtividade média de $3352 \mathrm{~kg} \mathrm{ha}^{-1}$. Para silagem, a produtividade média do milho está em torno de 20 a $30 \mathrm{t} \mathrm{ha}^{-1}$ de massa verde ( 8 a $12 \mathrm{t} \mathrm{ha}^{-1}$ de massa seca), podendo atingir cifras acima de $20 \mathrm{t} \mathrm{ha}^{-1}$ de massa seca, dependendo da fertilidade do solo, da cultivar e do clima (SAWAZAKi et al., 1998; CRUZ e Pereira Filho, 2001).

Em termos pedoedafológicos a granulometria do solo é um dos atributos físicos mais importantes para o desenvolvimento da cultura do milho, uma vez que exerce considerável influência sobre a capacidade de retenção e disponibilidade de água e de nutrientes do solo (Olson e SANDER, 1988). Outras propriedades físicas, como a consistência, estabilidade dos agregados, infiltração e aeração, também são bastante afetadas pela granulometria do solo, promovendo, no geral, uma diminuição relativamente considerável da produtividade vegetal (KIEHL, 1979).

O uso da geoestatística, como ferramenta de estudo da variabilidade dos atributos do solo, tem se mostrado de grande importância na interpretação e compreensão de resultados, com base na estrutura da sua variabilidade natural, principalmente da interação entre os atributos físicos do solo e a produtividade das culturas (ARAújo, 2002). Atualmente, tem sido constatado grande incremento da pesquisa da variabilidade espacial voltada à granulometria do solo e à produtividade vegetal como, por exemplo, os trabalhos de Kitamura (2004), MonteZano et al. (2006).

Desta forma, a presente pesquisa objetivou analisar a variabilidade e a dependência espacial dos atributos estudados da planta e do solo, bem como, as correlações lineares e espaciais entre a produtividade de forragem do milho e as frações granulométricas do solo pesquisado.

O experimento foi desenvolvido em $2005 \mathrm{em}$ Pereira Barreto (SP), latitude $20^{\circ} 40^{\prime} 12^{\prime \prime}$ S e longitude $51^{\circ} 01^{\prime} 50^{\prime \prime}$ W. O tipo climático é Aw (Koeppen) com precipitação pluvial e temperatura médias anuais de $1300 \mathrm{~mm}$ e $24,1^{\circ} \mathrm{C}$ respectivamente. O solo estudado foi Latossolo Vermelho Distrófico típico, textura francoarenosa, álico (EMBRAPA, 2006b).

A planta trabalhada foi o milho safrinha outonal (Zea mays L.) cuja semeadura ocorreu sobre pastagem (Brachiaria brizanta, cv Marandu), dessecando-a com glyphosate $\left(1,8 \mathrm{~kg} \mathrm{ha}^{-1}\right.$ p.a.). O híbrido simples utilizado foi o 30F80 semeado em 10/ 2/05, no sistema plantio direto, em área irrigada por pivô central. Seguiram-se as instruções agrícolas, conforme SAWAZAKI et al. (1998), tendo por base a análise inicial da fertilidade do solo, realizada em 3 / $2 / 05$. O espaçamento entre linhas foi de $0,85 \mathrm{~m}$, com uma densidade de 5,5 sementes $/ \mathrm{m}^{-1}$ na linha. $\mathrm{Na}$ semeadura utilizou-se $320 \mathrm{~kg} \mathrm{ha}^{-1}$ da fórmula 08-2816 (N-P-K). Na cobertura foram feitas duas aplicações de uréia (28/2/05 e 16/3/05), ambas na dosagem de $106 \mathrm{~kg} \mathrm{ha}^{-1}$, e uma aplicação de cloreto de potássio $\left(150 \mathrm{~kg} \mathrm{ha}^{-1}\right)$ em 3/3/2005.

A malha de amostragem de dados, definida numa área do referido pivô entre dois terraços agrícolas com declividade média de $0,025 \mathrm{~m} / \mathrm{m}$, ficou constituída com um total de 125 pontos (76 referente à grande malha, com espaçamento de $5 \mathrm{~m}$ entre pontos, e 49 referente à malha fina, com espaçamento de $1 \mathrm{~m}$ entre pontos), distribuídos em uma área de $2500 \mathrm{~m}^{2}$ (50 m x $50 \mathrm{~m}$ ). As áreas úteis para a de coleta de dados (do solo e planta), ficaram assim estabelecidas: a) na grande malha, uma área de $11,56 \mathrm{~m}^{2}(3,40 \mathrm{~m} \times 3,40 \mathrm{~m})$, e b) na malha fina, uma área de $2,55 \mathrm{~m}^{2}(2,55 \mathrm{~m} \mathrm{x} 1,00 \mathrm{~m})$.

Os atributos da granulometria foram areia (AR), silte (SI) e argila (AG), coletados nas profundidades de 0,00-0,10 m; 0,10-0,20 m e 0,20-0,30 $\mathrm{m}$, em $1 / 5 / 05$, analisados pela metodologia da pipeta, utilizando-se como dispersante o NaOH $1 \mathrm{~N}$ (EMBRAPA, 1997). Em função da profundidade de coleta, os atributos granulométricos foram identificados como: a) de 0,00-0,10 m: AR1, SI1 e AG1; b) de 0,10-0,20 m: AR2, SI2 e AG2, e c) de 0,20-0,30 m: AR3, SI3 e AG3.

$\mathrm{O}$ atributo da planta coletado em 30/4/05 foi a produtividade de massa verde de milho, cujo estágio fenológico estava entre R4 e R5. Posteriormente, transformou-se para massa seca da forragem (MSF), pela secagem em estufa, a $65^{\circ} \mathrm{C}$, até obtenção da massa constante. A expressão para o cálculo do atributo da planta (MSF), para cada ponto amostral, foi dada por:

$$
\mathrm{MSF}=(\mathrm{PU} / \mathrm{AP}) . \mathrm{FC} \cdot 10^{4}
$$

em que MSF = produtividade de massa seca num determinado ponto amostral $\left(\mathrm{kg} \mathrm{ha}^{-1}\right)$; $\mathrm{PU}=$ massa úmida total das plantas contidas na área de cada ponto $(\mathrm{kg}) ; \mathrm{AP}=$ área útil do ponto amostral $\left(\mathrm{m}^{2}\right) ; \mathrm{FC}=$ fator de correção, de massa verde para massa seca, calculada a partir de 10 plantas tomadas ao acaso dentro de cada ponto amostral, e $10^{4}=$ fator de conversão de $\mathrm{kg} \mathrm{m}^{-2}$ para $\mathrm{kg} \mathrm{ha}^{-1}$.

Efetuou-se a análise descritiva clássica por meio do pacote estatístico SAS (SCHLOTZHAVER e LitTell, 1997), e o teste de normalidade conforme SHAPIRO e WiLK (1965) a 1\%. Foi montada uma matriz de correlação objetivando efetuar as correlações lineares entre as combinações, duas a duas, entre todos os atributos estudados. 
$\mathrm{Na}$ análise geoestatística, utilizou-se o Gamma Design Software 7.0 (Gs+, 2004). Aos atributos com tendência em seus dados, aplicou-se a técnica do refinamento conforme SILVA et al. (2003). Os ajustes semivariográficos simples e cruzados e a decisão sobre seus modelos foram efetuados preferencialmente pela seleção do menor valor da soma dos quadrados dos desvios e pela análise da validação cruzada. A análise da dependência espacial (ADE), conforme KitAmURA et al. (2007), foi: $\mathrm{ADE}=25 \%$ (fraca); $25 \%<\mathrm{ADE}=75 \%$ (moderada); e ADE > 75\% (forte).

Conforme a classificação de Pimentel-Gómez (2002), na tabela 1, a MSF foi de média variabilidade concordando com os dados de Nussio (1991). Já para a granulometria do solo, SI1, SI3, AG1, AG2 e AG3, havia média variabilidade, ao passo que no resto dos atributos foi baixa. Assim, de forma geral, esses dados ficaram em consonância com os de Gonçalves e Folegatti (2002), Kitamura (2007), SouZa et al. (2004) e Montezano et al. (2006).

A granulometria dos latossolos é um atributo praticamente estável, dependendo pouco do uso e manejo do solo, e com variabilidade proveniente da sua formação natural (SouzA et al., 2004). Assim, os coeficientes de variação (Tabela 1) tiveram baixa magnitude e pequena variação entre as três camadas para AR, SI, e AG, semelhante aos dados de KitAmURA
(2007). A exceção ficou para SI2 com seus valores substituídos pelos seus respectivos logaritmos decimais. A distribuição de frequência para MSF foi normal, com valor médio de $14842 \mathrm{~kg} \mathrm{ha}^{-1}$, ficando tal cifra dentro da faixa de produtividade indicada por Cruz e Pereira Filho (2001). Contudo, essa produtividade foi substancialmente superior à relatada por SAWAZAKI et al. (1998) para o Estado de São Paulo (8000-12000 kg ha-1), provavelmente devido à irrigação utilizada.

Para a granulometria (Tabela 1), a distribuição de frequência foi normal (AR1 e AR2), e indeterminada para AR3, semelhante aos dados de Kitamura (2004). Seus valores médios foram de 804 $\mathrm{g} \mathrm{kg}^{-1}$ (AR1) a $763 \mathrm{~g} \mathrm{~kg}^{-1}$ (AR3) com tendência linear negativa em relação ao aumento da profundidade do solo. Para o silte, a distribuição foi normal (SI1 e SI3), ao passo que em SI2 (l-SI2) notou-se distribuição tendendo à lognormal. Suas médias foram de $90 \mathrm{~g} \mathrm{~kg}^{-1}$ (SI1), $82 \mathrm{~g} \mathrm{~kg}^{-1}$ (l-SI2) e $85 \mathrm{~g} \mathrm{~kg}^{-1}$ (SI3). Para argila, a distribuição foi normal (AG1 e AG2), e indeterminada para AG3, concordando com os dados de Souza et al. (2004). Seus valores médios variaram de $106 \mathrm{~g} \mathrm{~kg}^{-}$ 1 (AG1) a $152 \mathrm{~g} \mathrm{~kg}^{-1}$ (AG3), aumentando gradativamente com o aumento da profundidade do perfil do solo, concordando com os trabalhos de KitAmura (2004) e Souza et al. (2004).

Tabela 1. Análise descritiva inicial da produtividade de MSF de milho e das frações granulométricas de um Latossolo Vermelho Distrófico de Pereira Barreto (SP)

\begin{tabular}{|c|c|c|c|c|c|c|c|c|c|c|}
\hline \multirow{4}{*}{ Atributo } & \multicolumn{10}{|c|}{ Medidas estatísticas descritivas } \\
\hline & \multirow[t]{3}{*}{ Média } & \multirow[t]{3}{*}{ Mediana } & \multicolumn{2}{|c|}{ Valor } & \multirow{3}{*}{$\begin{array}{l}\text { Desvio } \\
\text { Padrão }\end{array}$} & \multicolumn{3}{|c|}{ Coeficiente } & \multirow{2}{*}{\multicolumn{2}{|c|}{$\begin{array}{c}\text { Probabilidade } \\
\text { do teste }\end{array}$}} \\
\hline & & & \multirow{2}{*}{ Mínimo } & \multirow{2}{*}{ Máximo } & & \multirow{2}{*}{ Variação } & \multirow{2}{*}{ Curtose } & \multirow{2}{*}{ Assimetria } & & \\
\hline & & & & & & & & & $\operatorname{Pr}<W$ & DF \\
\hline & & & & & + & & & & & \\
\hline & & & & & Atribut & da planta & & & & \\
\hline \multirow[t]{2}{*}{ MSF (kg/ha) } & 14842 & 15050 & 8949 & 23198 & 2424 & 16,3 & 0,615 & 0,008 & 0,369 & $\mathrm{NO}$ \\
\hline & \multicolumn{10}{|c|}{ Granulometria do solo } \\
\hline AR1 (g/kg) & 804 & 805 & 754 & 846 & 20,6 & 2,6 & $-0,582$ & 0,010 & 0,310 & $\mathrm{NO}$ \\
\hline AR2 (g/kg) & 787 & 786 & 734 & 841 & 20,6 & 2,6 & 0,003 & 0,092 & 0,967 & NO \\
\hline AR3 (g/kg) & 763 & 761 & 718 & 827 & 20,8 & 2,7 & 1,536 & 0,814 & $1,000.10^{-4}$ & IN \\
\hline SI1 (g/kg) & 90 & 90 & 56 & 147 & 16,3 & 18,0 & 0,258 & 0,429 & 0,192 & $\mathrm{NO}$ \\
\hline l-SI2 (g/kg) & 81 & 82 & 54 & 114 & $7,9.10^{-2}$ & 4,1 & $-0,897$ & $-0,040$ & 0,030 & $\mathrm{TL}$ \\
\hline SI3 (g/kg) & 85 & 84 & 49 & 128 & 15,0 & 17,7 & $-0,058$ & 0,203 & 0,821 & $\mathrm{NO}$ \\
\hline AG1 (g/kg) & 106 & 106 & 60 & 162 & 15,0 & 14,0 & 1,535 & 0,051 & 0,070 & $\mathrm{NO}$ \\
\hline AG2 (g/kg) & 131 & 130 & 89 & 171 & 16,2 & 12,4 & 0,278 & 0,038 & 0,173 & $\mathrm{NO}$ \\
\hline AG3 (g/kg) & 152 & 153 & 103 & 187 & 16,9 & 11,1 & 0,509 & $-0,638$ & 0,008 & IN \\
\hline
\end{tabular}

MSF = produtividade da massa seca da forragem do milho; AR, SI e AG são respectivamente as frações areia, silte e argila do solo; DF = distribuição de freqüência, sendo NO, TL e IN respectivamente do tipo normal, tendendo a lognormal e indeterminado, valores $x^{*}$ da média, mediana, mínimo e máximo retrotransformados por $\mathrm{x}^{*}=10 \log \mathrm{x}$. 
Na tabela 2, as correlações lineares entre o atributo da planta e os do solo foram todas baixas, diferentemente do observado por MonTEZANo et al. (2006). A única correlação significativa foi para o par MSF x AG1 $\left(r=-0,144^{*}\right)$ que, apesar de estatisticamente significativo, possivelmente devido ao grande número de amostras, revelou um coeficiente de correlação (r) muito baixo. Porém, ressalta-se que a irrigação possibilitou condições ótimas de disponibilidade de água à cultura do milho e, muito possivelmente, tal fato pode ter mascarado a interação entre os dados do solo e da planta em relação às condições naturais de entressafra.

Tabela 2. Matriz de correlação linear entre a produtividade de MSF de milho e as frações granulométricas de um Latossolo Vermelho Distrófico de Pereira Barreto (SP)

\begin{tabular}{|c|c|c|c|c|c|c|c|c|c|}
\hline \multirow{2}{*}{ Atributos } & \multicolumn{9}{|c|}{ Coeficiente de correlação } \\
\hline & MSF & AR1 & AR2 & AR3 & SI1 & l-SI2 & SI3 & AG1 & AG2 \\
\hline AR1 & $0,076 \mathrm{~ns}$ & - & - & - & - & - & - & - & - \\
\hline AR2 & $0,037 \mathrm{~ns}$ & $0,616^{* *}$ & - & - & - & - & - & - & - \\
\hline AR3 & $0,015 \mathrm{~ns}$ & $0,470^{* *}$ & $0,683^{* *}$ & - & - & - & - & - & - \\
\hline SI1 & $0,035 \mathrm{~ns}$ & $-0,699 * *$ & $-0,357^{* *}$ & $-0,178^{* *}$ & - & - & - & - & - \\
\hline$l$-SI2 & $-0,056 \mathrm{~ns}$ & $-0,563^{* *}$ & $-0,627^{* *}$ & $-0,490^{* *}$ & $0,479^{* *}$ & - & - & - & - \\
\hline SI3 & $-0,057 \mathrm{~ns}$ & $-0,402^{* *}$ & $-0,418^{* *}$ & $-0,587^{* *}$ & $0,421^{* *}$ & $0,664^{* *}$ & - & - & - \\
\hline AG1 & $-0,144^{*}$ & $-0,619^{* *}$ & $-0,463^{* *}$ & $-0,456^{* *}$ & $-0,128^{*}$ & $0,255^{* *}$ & $0,095 \mathrm{~ns}$ & - & - \\
\hline AG2 & $0,011 \mathrm{~ns}$ & $-0,260^{* *}$ & $-0,700^{* *}$ & $-0,422^{* *}$ & $0,005 \mathrm{~ns}$ & $-0,114 \mathrm{~ns}$ & $-0,076 \mathrm{~ns}$ & $0,356^{* *}$ & - \\
\hline AG3 & $0,066 \mathrm{~ns}$ & $-0,238^{* *}$ & $-0,487^{* *}$ & $-0,658^{* *}$ & $-0,117 \mathrm{~ns}$ & $0,041 \mathrm{~ns}$ & $-0,107 \mathrm{~ns}$ & $0,459^{* *}$ & $0,585^{* *}$ \\
\hline
\end{tabular}

MSF é a produtividade de massa seca da forragem do milho; AR, SI e AG, são respectivamente as frações de areia, silte e argila; atributo precedido de " $l$ " foi transformado pelo logaritmo decimal; * significativo ao nível de $5 \%$ de probabilidade; ${ }^{* *}$ significativo ao nível de $1 \%$ de probabilidade, e ns não significativo.

Já para as correlações entre os atributos granulométricos (Tabela 2), observou-se que as correlações estabelecidas para as mesmas camadas, entre os pares dos atributos ARxSI e ARxAG, foram todas de forma negativas e cientificamente coerentes, fato observado parcialmente por Kitamura (2004) e Montezano et al. (2006). As mais expressivas correlações observadas nas diferentes camadas do solo foram: a) AR1 x SI1 (r= -0,699**) de 0,00-0,10 m; b) AR2 $x$ AG2 $\left(r=-0,700^{* *}\right)$ de $0,10-0,20 \mathrm{~m}$, e c) AR3 x AG3 (r $\left.=-0,658^{* *}\right)$ de $0,20-0,30 \mathrm{~m}$.

Na tabela 3, na análise geoestatística, para os ajustes semivariográficos simples, à exceção do $r$-SI1, os demais atributos tiveram dependência espacial. Para ajuste da MSF o coeficiente de determinação espacial foi de 0,770 , enquanto para a granulometria, variaram de 0,599 (AG3) a 0,912 (l-SI2). O grau de dependência espacial da MSF foi moderada (73,5\%). Deste modo, $73,5 \%$ da variação da produtividade de MSF do milho foram explicadas pela dependência espacial. Para demais atributos o grau de dependência foi entre $53,1 \%$ (r-AR1) e $93,1 \%$ (AG3), concordando com os dados de Gonçalves e Folegatti (2002), Kitamura (2004) e Souza et al. (2004), em se puderam notar classes de dependência espacial ora moderada ora alta.
Os modelos semivariográficos (Tabela 3 ) foram o exponencial (MSF, $r$-AR1, $l$-SI2, $r$-SI3) e o esférico (AR2, AR3, AG1, AG2, AG3), semelhante aos de Gonçalves e Folegatti (2002) e Kitamura (2004), onde tais modelos foram obtidos. Já o alcance da dependência variou de 8,5 m (AG3) a 46,8 m (l-SI2), semelhante aos de GonÇALVES et al. (2001) e EGUCHI et al. (2002).

$\mathrm{Na}$ análise geoestatística das cokrigagens (Tabela 3), o único ajuste semivariográfico cruzado foi $\mathrm{o} \mathrm{MSF}=\mathrm{f}(\mathrm{AG} 3)$, que apesar dos lags em dois quadrantes do semivariograma (Figura 1a), denotando, conforme LimA (2007), uma relativa indefinição na forma como a variável secundária atua, se positiva ou negativamente a curtos alcances, constatou-se moderado coeficiente de dependência espacial $\left(\mathrm{r}^{2}=0,506\right)$. O modelo de ajuste foi o esférico, com alcance de 29,6 m. Desta forma, a MSF do milho pode ser espacialmente estimada por meio da fração argila de 0,20-0,30m (AG3) conforme o mapa de cokrigagem $[\mathrm{MSF}=\mathrm{f}(\mathrm{AG} 3)]$ apresentado na Figura $1 \mathrm{~b}$.

Nos atributos estudados há variabilidade dos dados entre média e baixa. no âmbito espacial não variam aleatoriamente, revelando padrões de dependência bem definidos. 
Tabela 3. Parâmetros dos semivariogramas ajustados para a produtividade de MSF de milho e para as frações granulométricas de um Latossolo Vermelho Distrófico de Pereira Barreto (SP)

\begin{tabular}{|c|c|c|c|c|c|c|c|c|}
\hline \multirow[b]{2}{*}{ Atributo } & \multicolumn{7}{|c|}{ Parâmetros } & \multirow[b]{2}{*}{$\mathrm{ADE}$} \\
\hline & Modelo & $\begin{array}{c}\text { Efeito } \\
\text { Pepita (C0) }\end{array}$ & $\begin{array}{r}\text { Patamar } \\
(\mathrm{C} 0+\mathrm{C})\end{array}$ & $\begin{array}{c}\text { Variância } \\
\text { Estrutural (C) }\end{array}$ & $\begin{array}{c}\text { Alcance } \\
(\mathrm{m})\end{array}$ & $\mathrm{r} 2$ & SQR & \\
\hline \multicolumn{9}{|c|}{$\gamma(h)$ simples do atributo da planta } \\
\hline MSF (kg/ha) & $\exp$ & $1,250.106$ & $4,715.106$ & $3,465.106$ & 11,8 & 0,770 & $1,667.1012$ & 73,5 \\
\hline \multicolumn{9}{|c|}{$\gamma(h)$ simples dos atributos granulométricos do solo } \\
\hline$r$-AR1 (g/kg) & $\exp$ & $1,509.102$ & $3,218.102$ & $1,709.102$ & 38,4 & 0,808 & $2,489.103$ & 53,1 \\
\hline $\operatorname{AR2}(\mathrm{g} / \mathrm{kg})$ & esf & $1,050.102$ & $4,577.102$ & $3,527.102$ & 17,4 & 0,882 & $1,672.104$ & 77,1 \\
\hline AR3 (g/kg) & esf & $1,020.102$ & $4,266.102$ & $3,246.102$ & 15,4 & 0,811 & $2,121.104$ & 76,1 \\
\hline$r-\mathrm{SI} 1(\mathrm{~g} / \mathrm{kg})$ & epp & $2,019.102$ & $2,019.102$ & - & - & - & - & - \\
\hline l-SI2 (g/kg) & $\exp$ & $2,070.10-3$ & $7,280.10-3$ & $5,210.10-3$ & 46,8 & 0,912 & $1,459.10-6$ & 71,6 \\
\hline$r$-SI3 (g/kg) & $\exp$ & $8,610.101$ & $1,875.102$ & $1,014.102$ & 30,3 & 0,734 & $1,418.103$ & 54,1 \\
\hline AG1 (g/kg) & esf & $2,810.101$ & $1,959.102$ & $1,678.102$ & 12,8 & 0,827 & $3,295.103$ & 82,7 \\
\hline AG2 (g/kg) & esf & $2,350.101$ & $2,400.102$ & 2.165 .102 & 13,7 & 0,720 & $1,310.104$ & 90,2 \\
\hline AG3 (g/kg) & esf & $1,710.101$ & $2,479.102$ & $2,308.102$ & 8,5 & 0,599 & $2,171.104$ & 93,1 \\
\hline \multicolumn{9}{|c|}{$\gamma(h)$ cruzado entre atributos } \\
\hline $\mathrm{MSF}=\mathrm{f}(\mathrm{AG} 3)$ & esf & $1,000.101$ & $6,489.103$ & $6,479.103$ & 29,6 & 0,506 & $4,830.107$ & 99,8 \\
\hline
\end{tabular}

MSF = produtividade da massa seca da forragem do milho; AR, SI e AG, são respectivamente as frações areia, silte e argila do solo; $\left({ }^{b}\right)$ exp. $=$ exponencial, esf.= esférico e epp.= efeito pepita puro; $\mathrm{SQR}=$ soma dos quadrados dos resíduos; $\mathrm{ADE}=$ avaliador da dependência espacial; atributos precedidos de " $l$ " e " $r$ " tiveram respectivamente valores representados pelos logaritmos decimais e pelos resíduos.
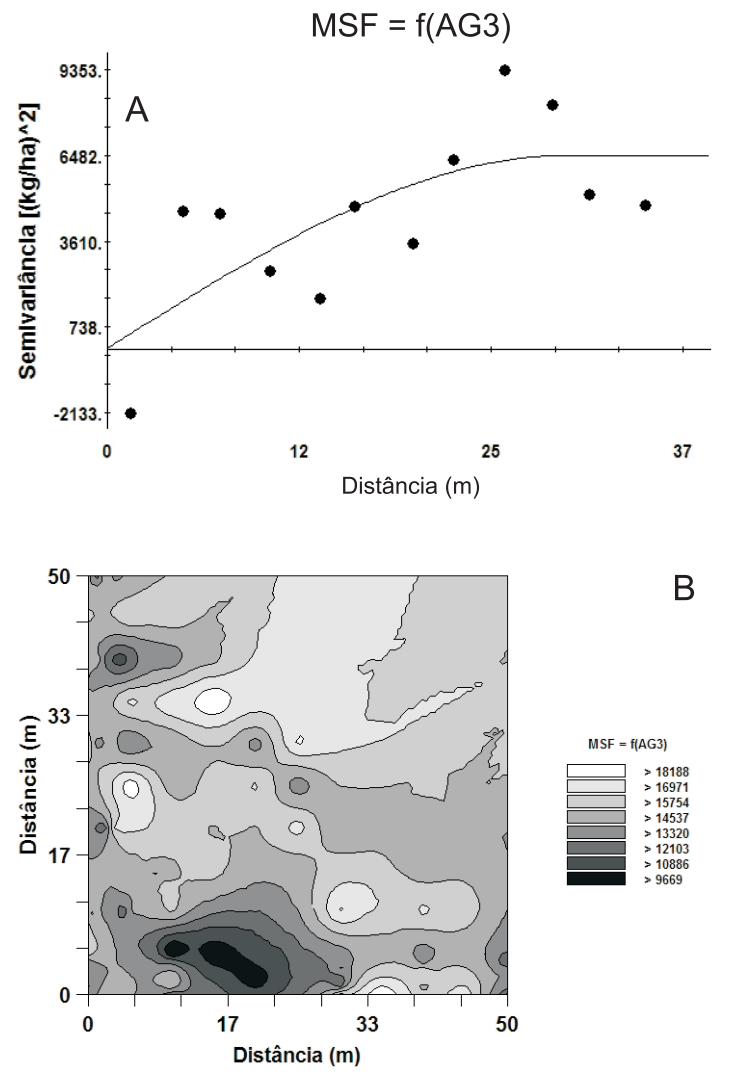

Figura 1. Semivariograma e mapa de cokrigagem da produtividade de MSF de milho em função da fração argila (AG3) de um Latossolo Vermelho Distrófico de Pereira Barreto (SP).
Não há correlação linear consistente entre a produtividade de forragem do milho (MSF) e as frações granulométricas, possivelmente devido à irrigação. Nota-se, porém, moderada correlação espacial, entre causa/efeito, para o par de atributos MSF e AG3.

\section{Referências}

ARAUJO, A.V. Variabilidade espacial de propriedades químicas e granulométricas do solo na definição de zonas homogêneas de manejo. 2002. 80f. Dissertação (Mestrado em Agronomia) - Faculdade de Ciências Agrárias e VeterináriasUNESP, Jaboticabal.

CRUZ, J.C.; PEREIRA FILHO, I.A. Cultivares de milho para silagem. In: CRUZ, J.C.; PEREIRA FILHO, I.A.; RODRIGUES, J.A.; FERREIRA, J.J. (Ed.). Produção e utilização de silagem de milho e sorgo. Sete Lagoas: Embrapa Milho e Sorgo, 2001. p.11-37.

EGUCHI, E.S.; SILVA, E.L.; OLIVEIRA, M.S. Variabilidade espacial da textura e da densidade de partículas em um solo aluvial no município de Lavras, MG. Revista Brasileira de Engenharia Agrícola Ambiental, v.6, p.242-246, 2002.

EMPRESA BRASILEIRA DE PESQUISA AGROPECUÁRIA EMBRAPA MILHO E SORGO. Introdução e importância econômica do milho. Disponível em: <http:// www.cnpms.embrapa.br/publicações/milhoesorgo $>$. Acesso em: Dez. 2006a. 
EMPRESA BRASILEIRA DE PESQUISA AGROPECUÁRIA EMBRAPA. Sistema brasileiro de classificação de solos. 2.ed. Rio de Janeiro: Embrapa, 2006b. 306 p.

EMPRESA BRASILEIRA DE PESQUISA AGROPECUÁRIA EMBRAPA. Manual de métodos de análise do solo. 2.ed. Rio de Janeiro: Embrapa, 1997. (numeração capitulada)

GONÇALVES, A.C.A.; FOLEGATTI, M.V. Correlação espacial entre retenção de água e textura do solo para fins de manejo de irrigação. Engenharia Agrícola, v.22, p.296-303, 2002.

GONÇALVES, A.C.A.; FOLEGATTI, M.V.; MATA, J.D.V. Análises exploratórias e geoestatística da variabilidade de propriedades físicas de um Argissolo Vermelho. Maringá, v.23, p.1149-1157, 2001.

GS+: Geostatistics for environmental sciences. 7.ed. Michigan, Plainwell: Gamma Desing Software, 2004. 159p.

KIEHL, E.J. Manual de edafologia: relações solo-planta. Piracicaba: Agronômica Ceres, 1979. 264 p.

KITAMURA, A.E.; CARVALHO, M.P.; LIMA, C.G.R. Relação entre a variabilidade espacial das frações granulométricas do solo e a produtividade do feijoeiro sob plantio direto. Revista Brasileira de Ciência do Solo, v.31, p.361-369, 2007.

LIMA, C.G.R. Correlação linear e espacial da produtividade de forragem de milho com atributos físicos de um latossolo vermelho distrófico. 2007. 107f. Dissertação (Mestrado em Agronomia) - Faculdade de Engenharia-UNESP, Ilha Solteira.

MONTEZANO, Z.F.; CORAZZA, E.J.; MURAOKA, T. Variabilidade espacial da fertilidade do solo em área cultivada e manejada homogeneamente. Revista Brasileira de Ciência do Solo, v.30, p.839-847, 2006.

NUSSIO, L.G. Cultura de milho para produção de silagem de alto valor alimentício. In: $4^{\circ}$ SIMPÓSIO SOBRE NUTRIÇÃO DE BOVINOS. 4.,1991, Piracicaba. Anais... Piracicaba: Fealq, 1991. p.58-168.

OLSON, R.A.; SANDER, D.H. Maize production. In: SPRAGUE, G.F.; DUDLEY, J.W. (Ed.). Corn and corn improvement. Madison: American Society of Agronomy, 1988. Cap 11, p.639-686.

PIMENTEL-GOMEZ, F.; GARCIA, C.H. Estatística aplicada a experimentos agronômicos e florestais: exposição com exemplos e orientações para uso de aplicativos. Piracicaba: Fealq, 2002. 309p.

SAWAZAKI, E.; GALVAO, J.C.C.; PATERNIANI, M.E.A.G.Z. Milho: Instruções técnicas. In: FAHL, J.I.; CAMARGO, M.B.P.; PIZZINATO, M.A.; BETTI, J.A.; MELO, A.M.T.; DE MARIA, I.C.; FURLANI, A.M.C. (Org.). Instruções agrícolas para as principais culturas econômicas (Boletim 200). 6.ed. Campinas: Instituto Agronômico, 1998, v.1, p.37-39.

SCHLOTZHAVER, S.D.; LITTELL, R.C. SAS: System for elementary statistical analysis. 2.ed. Cary: SAS institute, 1997. 905p.
SHAPIRO, S.S.; WILK, M.B. An analysis of variance test for normality: complete samples. Biometrika, v.52, p.591-611, 1965.

SILVA, V.R. et al. Variabilidade espacial das características químicas do solo e produtividade de milho em um Argissolo Vermelho-Amarelo Distroférrico Arênico. Revista Brasileira de Ciência do Solo, v.27, p.1013-1020, 2003.

SOUZA, Z.M.; Marques Júnior, J.; Pereira, G.T.; Barbieri, D.M.. Variabilidade espacial da textura de um Latossolo Vermelho eutroférrico sob cultivo de cana-de-açúcar. Engenharia Agrícola, v.24, p.309-319, 2004. 